\title{
CHAOS AND HIGHER ORDER DIFFERENCES
}

\author{
JOHN W. NEUBERGER
}

\begin{abstract}
Asymptotic doubling of magnitude of higher order differences of a function is linked with abrupt character changes in the function.
\end{abstract}

1. Introduction and statement of results. Suppose that $J$ is a connected open subset of the real numbers. If $\delta>0, u \in J, n$ is a positive integer, and $f$ is a continuous real-valued function on $J$, then

$$
\Delta_{f}(n ; u, \delta) \equiv \sum_{k=0}^{n}\left(\begin{array}{l}
n \\
k
\end{array}\right)(-1)^{n-k} f(u+k \delta)
$$

provided that $v=u+n \delta \in J$. For each such $u, n, \delta$ one has the natural upper bound

$$
\left|\Delta_{f}(n ; u, \delta)\right| \leqslant 2^{n}|f|_{[u, v]}
$$

i.e., $\left|\Delta_{f}(n ; u, \delta)\right|^{1 / n} \leqslant 2|f|_{[u, v]}^{1 / n}$ since $\sum_{k=0}^{n}\left(\begin{array}{c}n \\ k\end{array}\right)=2^{n}$.

In this note we consider the implications of this last inequality being asymptotically close to equality. To be more precise, denote by $G$ the collection of all bounded open intervals $S$ such that $\bar{S} \subset J$. For $f$ a continuous function on $J, S \in G$, and $\delta>0$ so that $\delta<|S|$,

$$
\Phi(S, \delta, f) \equiv \sup \left\{\left|\Delta_{f}(n ; u, \delta)\right|^{1 / n}: u, u+n \delta \in S, n \in Z^{+}\right\} .
$$

Definition. The statement that $f$ is chaotic at $x \in J$ means that if $S \in G$ and $x \in S$, then

$$
\lim _{\delta \rightarrow 0} \Phi(S, \delta, f)=2 .
$$

The following theorem helps place this definition into perspective.

THEOREM 1. If $S \in G$, then $\lim \sup _{\delta \rightarrow 0} \Phi(S, \delta, f) \leqslant 2$.

The definition is thus intended to describe certain extreme asymptotic behavior of higher order differences. It turns out that differences of a function which is chaotic at a point show a near doubling of size with increasing order of differences. Connections with work of Feigenbaum [2] seem to be of possible interest.

Received by the editors July 11, 1985. Presented to the Society, New Orleans, January 1986.

1980 Mathematics Subject Classification (1985 Revision). Primary 26E10; Secondary 42A55, 94A15, $58 \mathrm{~F} 14$.

Key words and phrases. Chaos, quasi-analyticity, higher order differences.

(C)1987 American Mathematical Society $0002-9939 / 87 \$ 1.00+\$ .25$ per page 
The following shows that there is a wide class of examples of chaotic functions.

THEOREM 2. Suppose $c \in J, g$ is a real-analytic function on $J$, and $f$ is a continuous function on $J$ such that

(i) $f(x)=g(x)$ if $x \in J$ and $x \leqslant c$, and

(ii) if $t>c$ there is $x$ in $(c, t)$ such that $f(x) \neq g(x)$.

Then $f$ is chaotic at $c$.

The following result of Beurling gives some additional perspective on the definition.

Theorem 3. Suppose $S \in G$ and $\lim _{\sup _{\delta \rightarrow 0}} \Phi(S, \delta, f)<2$. Then $f$ is real-analytic on $S$.

The following is essentially a converse to the above.

THEOREM 4. Suppose $f$ is real-analytic at $c \in J$. There are $M, \varepsilon>0$ such that if $S \in G, c \in S$, and $|S|<\varepsilon$, then

$$
\Phi(S, \delta, f) \leqslant|S| M \text { for all } \delta<|S| .
$$

The following indicates that differentiability is not necessary in order to have a nonchaotic function.

TheOrem 5. Suppose $J=R, 0<a<1, \lambda$ is an odd positive integer, $\lambda a \geqslant 1$, and

$$
f(x)=\sum_{k=0}^{\infty} a^{k} \cos \left(\lambda^{k} x\right), \quad x \in R .
$$

Then $f$ is nowhere differentiable and also nowhere chaotic.

Such a function $f$ is a Hardy-Weierstrass function (cf. [3]) which is known to be nowhere differentiable. On the other hand, if $f(x)=0, x \leqslant 0, f(x)=\exp \left(-x^{2}\right)$, $x>0$, then $f$ is infinitely differentiable yet chaotic at 0 , according to the following theorem (on which Theorem 2 is based) $[6,5]$.

THEOREM 6. Suppose $f$ is a continuous function on $[0,1]$ such that $f(x)=0$ for $x \in[0,1 / 2]$ and, if $t>1 / 2$, there is $x$ in $(1 / 2, t)$ such that $f(x) \neq 0$. Then if $a>0$,

$$
\lim _{\delta \rightarrow 0}\left(\sup _{1-a \leqslant n \delta \leqslant 1}\left|\Delta_{f}(n ; 0, \delta)\right|\right)^{\delta}=2 .
$$

Some readers may find it interesting to do computer calculations concerning the above result (and consequently Theorem 2). To this end we offer the following Basic code:

10 INPUT D, A

$20 \mathrm{Z}=0$

30 FOR L $=\operatorname{INT}((1-A) / D)$ TO INT(1/D)

$40 \mathrm{Y}=0$

$50 \mathrm{M}=1$

60 FOR $\mathrm{J}=0$ TO L

$70 \mathrm{X}=(\mathrm{L}-\mathrm{J}) * \mathrm{D}$ 


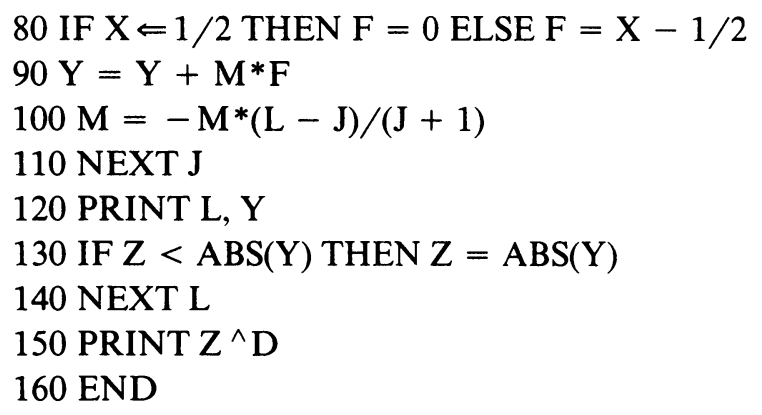

It is suggested that $D=.01, A=.1$ be tried. Line 80 contains a function definition and may be altered to compute with other functions.

\section{Proofs.}

Proof of Theorem 1. Suppose $S \in G, u \in S, \delta>0, n \in Z^{+}$, and $u+n \delta \in S$. Then

$$
\begin{aligned}
\left|\Delta_{f}(n ; u, \delta)\right| & =\left|\sum_{k=0}^{n}\left(\begin{array}{l}
n \\
k
\end{array}\right)(-1)^{n-k} f(u+k \delta)\right| \\
& \leqslant \sum_{k=0}^{n}\left(\begin{array}{l}
n \\
k
\end{array}\right)|f(u+k \delta)| \leqslant 2^{n}|f|_{S}
\end{aligned}
$$

and so

$$
\left|\Delta_{f}(n ; u, \delta)\right|^{1 / n} \leqslant 2|f|_{s}^{1 / n}
$$

Hence

$$
\Phi(S, \delta, f)=\sup \left\{\left|\Delta_{f}(n ; u, \delta)\right|^{1 / n}: u, u+n \delta \in S, n \in Z^{+}\right\} \leqslant 2 c^{\delta},
$$

where $c \equiv \max \left(1,|f|_{S}^{1 /|S|}\right)$. The conclusion follows since $\lim _{\delta \rightarrow 0} c^{\delta}=1$.

Proof of Theorem 4. Suppose $f$ is real-analytic at $c \in J$. Pick $r, q \in R$ such that $0<q<r$ and $r$ is less than the radius of convergence of a power series expansion of $f$ about $c$. Denote by $L, \rho$ positive numbers such that $\left|f^{(k)}(\alpha)\right| \leqslant L k ! \rho^{k}$ for all $\alpha \in[c-q, c+q], k \in Z^{+}$. Suppose also that $S \in G, c \in S,|S|<q$, $n \in Z^{+}, \delta>0$, and $u, u+n \delta \in S$. Then

$$
\left|\Delta_{f}(n ; u, \delta)\right|=\left|\left(\Delta_{f}(n ; u, \delta) / \delta^{n}\right) \delta^{n}\right|=\left|f^{(n)}(\alpha)\right| \delta^{n}
$$

for some $\alpha \in[u, u+n \delta]$. Hence

$$
\left|\Delta_{f}(n ; u, \delta)\right| \leqslant L n ! \rho^{n} \delta^{n} \leqslant L n^{n} \rho^{n} \delta^{n} \leqslant|S|^{n}\left(\rho L^{1 / n}\right)^{n} \leqslant(|S| M)^{n},
$$

where $\beta \equiv \max (1, L), M \equiv \rho \beta$. Therefore

$$
\Phi(S, \delta, f) \leqslant|S| M \quad \text { if } 0<\delta<|S| .
$$

Proof of Theorem 2. Suppose $c \in J$ and that $f$ as given in Theorem 2 is not chaotic at $c$. Denote by $S_{1}$ a member of $G$ containing $c$ so that

$$
\liminf _{\delta \rightarrow 0} \Phi\left(S_{1}, \delta, f\right)=2-\varepsilon
$$


for some $\varepsilon>0$. Denote by $\delta_{1}, \delta_{2}, \ldots$ a decreasing sequence of positive numbers converging to 0 so that $\lim _{k \rightarrow \infty} \Phi\left(S_{1}, \delta_{k}, f\right)=2-\varepsilon$. Using Theorem 4 , there is $S_{2} \in G$ such that $S_{2}$ contains $c$ and $\Phi\left(S_{2}, \delta, g\right) \leqslant \varepsilon / 2$ if $0<\delta<\left|S_{2}\right|$. Define $h$ so that if $x \in J$ then

$$
h(x)= \begin{cases}0 & \text { for } x \leqslant c \\ f(x)-g(x) & \text { for } x>c .\end{cases}
$$

Note that if $t>x$ there is $x_{1}$ in $(x, t)$ so that $h\left(x_{1}\right) \neq 0$. Note also that $f(t)=h(t)$ $+g(t), t \in J$. Pick $r$ and $s$ in $S_{1} \cap S_{2}$ so that $r<c<s$ and $c=(r+s) / 2$. Define $w$ on $[0,1]$ so that $w(t)=h(t s+(1-t) r), t \in[0,1]$. Note that

$$
\Delta_{w}(n ; 0, \delta)=\Delta_{h}(n, r, \delta(s-r)), \quad n \in Z^{+}, 0<n \delta<1 .
$$

Suppose $a>0$ and note that by Theorem 5 ,

$$
\lim _{k \rightarrow \infty}\left(\sup _{1-a \leqslant n \beta_{k} \leqslant 1}\left|\Delta_{w}\left(n ; 0, \beta_{k}\right)\right|\right)^{\beta_{k}}=2,
$$

where $\beta_{k} \equiv \delta_{k} /(s-r), k \in Z^{+}$.

For each $k>0$, pick $n_{k}$ such that

$$
\left|\Delta_{w}\left(n_{k}, 0, \beta_{k}\right)\right|=\sup _{1-a \leqslant n \beta_{n} \leqslant 1}\left|\Delta_{w}\left(n ; 0, \beta_{k}\right)\right|
$$

and note that

$$
\left|\Delta_{w}\left(n_{k} ; 0, \beta_{k}\right)\right|^{\beta_{k}} \rightarrow 2 \text { as } k \rightarrow \infty .
$$

Since $\beta_{k} n_{k} \leqslant 1, k \in Z^{+}$, it follows that $1 / n_{k} \geqslant \beta_{k}, k \in Z^{+}$, and hence

$$
\left|\Delta_{w}\left(n_{k} ; 0, \beta_{k}\right)\right|^{1 / n_{k}} \rightarrow 2 \text { as } k \rightarrow \infty
$$

since

$$
\limsup _{k \rightarrow \infty}\left|\Delta_{w}\left(n_{k} ; 0, \beta_{k}\right)\right|^{\beta_{k}} \leqslant \limsup _{k \rightarrow \infty}\left|\Delta_{w}\left(n_{k} ; 0, \beta_{k}\right)\right|^{1 / n_{k}} \leqslant 2 .
$$

Therefore, $\left|\Delta_{h}\left(n_{k}, r, \delta_{k}\right)\right|^{1 / n_{k}} \rightarrow 2$ as $k \rightarrow \infty$. But

$$
\left|\Delta_{h}\left(n_{k} ; r, \delta_{k}\right)\right| \leqslant\left|\Delta_{f}\left(n_{k} ; r, \delta_{k}\right)\right|+\left|\Delta_{g}\left(n_{k} ; r, \delta_{k}\right)\right|
$$

and so

$$
\left|\Delta_{h}\left(n_{k} ; r, \delta_{k}\right)\right|^{1 / n_{k}} \leqslant\left(\left|\Delta_{f}\left(n_{k} ; r, \delta_{k}\right)\right|+\varepsilon / 2\right)^{1 / n_{k}} \text {. }
$$

Hence

$$
\limsup _{k \rightarrow \infty}\left|\Delta_{h}\left(n_{k} ; r, \delta_{k}\right)\right|^{1 / n_{k}} \leqslant 2-\varepsilon
$$

since

$$
\limsup _{k \rightarrow \infty} \Phi\left(S_{1} \cap S_{2}, \delta_{k}, f\right) \leqslant 2-\varepsilon, \quad \text { a contradiction. }
$$

Hence $f$ is chaotic at $c$ and the theorem is established. 
Proof of Theorem 5. Under the hypothesis, write

$$
f(x)=\operatorname{Re}\left(\sum_{k=0}^{\infty} a^{k} \exp \left(i \lambda^{k} x\right)\right), \quad x \in R .
$$

An easy calculation gives

$$
\Delta_{f}(n ; u, \delta)=\operatorname{Re}\left(\sum_{k=0}^{\infty} a^{k} \exp \left(i \lambda^{k} x\right)\left(\exp \left(i \lambda^{k} \delta\right)-1\right)^{n}\right)
$$

and hence

$$
\left|\Delta_{f}(n ; u, \delta)\right| \leqslant 2^{n} \sum_{k=0}^{\infty} a^{k}\left|\sin \left(\lambda^{k} \delta / 2\right)\right|^{n}, \quad u \in R, \delta>0, n \in Z^{+},
$$

using the identity $\exp (i \alpha)-1=2 i \exp (i \alpha / 2) \sin (\alpha / 2), \alpha \in R$. Choose $\delta_{q}=2 \pi / \lambda^{q}$, $q \in Z^{+}$. Clearly for $z, q \in Z^{+}, \lambda^{k} \delta_{q} / 2=\pi \lambda^{k-q}=(\pi / 2)\left(2 \lambda^{k-q}\right)$. Hence if $k-q \geqslant 0$ then $\lambda^{k} \delta_{q} / 2$ is an even multiple of $\pi / 2$ and so $\sin \left(\lambda^{k} \delta_{q} / 2\right)=0$. On the other hand if $k-q<0$ then $\lambda^{k} \delta_{q} / 2=(\pi / 2) 2 / \lambda^{q-k}$ and $2 / \lambda^{q-k}<2 / \lambda \leqslant 2 / 3$ and so $0<$ $\sin \left(\lambda^{n} \delta_{q} / 2\right) \leqslant \sin (\pi / 3)=\sqrt{3} / 2$. Hence

$$
\left|\Delta_{f}\left(n ; u, \delta_{q}\right)\right| \leqslant 2^{n} \sum_{k=0}^{\infty} a^{k}(\sqrt{3} / 2)^{n} \leqslant(\sqrt{3})^{n}(1-a)^{-1}, \quad n, q \in Z^{+} .
$$

Denote by $S$ a bounded open interval. Note that if $n, q \in Z^{+}$and $u, u+n \delta_{q} \in S$, then

$$
\left|\Delta_{f}\left(n ; u, \delta_{q}\right)\right|^{1 / n} \leqslant \sqrt{3}(1-a)^{-1 / n} .
$$

Suppose now that $\lim \sup _{q \rightarrow \infty} \Phi\left(S, \delta_{q}, f\right)=2$ and denote by $\left\{n_{q}\right\}_{q=1}^{\infty}$ a sequence in $Z^{+}$and by $\left\{u_{q}\right\}_{q=1}^{\infty}$ a sequence in $S$ so that $u_{q}+\delta_{q} n_{q} \in S, q \in S$, and

$$
\limsup _{q \rightarrow \infty}\left|\Delta_{f}\left(n_{q}, u_{q}, \delta_{q}\right)\right|^{1 / n_{q}}=2 \text {. }
$$

It must be that $n_{q} \rightarrow \infty$ as $q \rightarrow \infty$ for otherwise $\left\{\mid \Delta_{f}\left(n_{q}, u_{q}, \delta_{q}\right)\right\}_{q=1}^{\infty}$ would have a subsequence converging to 0 (using the continuity of $f$ ). Hence

$$
\left|\Delta_{f}\left(n_{q}, u_{q}, \delta_{q}\right)\right|^{1 / n_{q}} \leqslant \sqrt{3}(1-a)^{-1 / n_{4}} \rightarrow \sqrt{3} \quad \text { as } q \rightarrow \infty,
$$

a contradiction.

Therefore $\lim \sup _{q \rightarrow \infty} \Phi\left(S, \delta_{q}, f\right)<2$ and hence $f$ is not chaotic at any point of $S$. Hence $f$ is nowhere chaotic.

Theorem 6 is essentially Lemma 8 of [5]. In connection with Theorem 3 see [1, 6].

3. Notes and comments. This work is closely related to early work of this writer on quasi-analytic collections [6, 7]. A collection of continuous functions on an open set $J$ is called quasi-analytic if no two members of it agree on an open subset of $J$. It may be seen that a function which is nonchaotic at $c \in J$ is for some bounded open interval $S$ containing $c$ a member of the quasi-analytic collection $G(\sigma, S)$ (cf. [6]) where $\sigma=\left\{\delta_{q}\right\}_{q=1}^{\infty}$ so that $\limsup _{q \rightarrow \infty} \Phi\left(S, \delta_{q}, f\right)<2$. It is mentioned that all members of all the quasi-analytic collections defined in [6 and 7] are nonchaotic. 
For relationships between Theorems 3 and 5 with semigroups of operators, see [6, 4] as well as $[\mathbf{1 , 8}]$.

Near doubling of differences in chaotic situations may be illustrated by running the Basic code given above for various continuous functions $f$ which are zero on $[0,1 / 2]$ but not identically zero on any interval $[0, t], t>1 / 2$. Without a fairly careful analysis of roundoff error one may falsely conclude that certain nonchaotic functions are chaotic ( $\operatorname{try} y^{\prime}$ the sine function for example). In a sense, roundoff error sometimes induces a chaos of its own which is easily confused with the mathematical chaos defined in this note. In fact observation of false period doubling in computations with Feigenbaum's function $\left(f_{b}: f_{b}(x)=b x(1-x), x \in[0,1]\right.$; cf. [2]) led to the idea of connecting [5-7] with the study of chaos.

This writer is indebted to Walter Rudin for pointing out reference [3] and for his general comments concerning lacunary Fourier series.

Finally this writer offers the outright speculation that chaos as defined here (but extended to higher dimensional cases) may provide a mathematical expression, in certain instances, of the physical phenomena of turbulence.

\section{REFERENCES}

1. A. Beurling, On analytic extension of semigroups of operators, J. Funct. Anal. 6 (1970), 387-400.

2. M. Feigenbaum, Quantitative universality for a class of nonlinear transformations, J. Statist. Phys. 19 (1978), 25-52.

3. J.-P. Kahane, Lacunary Taylor and Fourier series, Bull. Amer. Math. Soc. 70 (1964), 199-213.

4. D. G. Kendall, Some recent developments in the theory of denumerable Markov processes, Trans. Fourth Prague Conference on Information Theory, Statistical Decision Functions, Random Processes (Prague 1965), Academia, Prague, 1967, pp. 11-27.

5. J. W. Neuberger, A quasi-analytic condition in terms of finite differences, Proc. London Math. Soc. (3) 14 (1964), 245-259.

6. __ Quasi-analyticity and semigroups, Bull. Amer. Math. Soc. 78 (1972), 909-922.

7. Q Quasi-analytic collections containing Fourier series which are not infinitely differentiable, J. London Math. Soc. 43 (1968), 612-616.

8. G. Piesier, Holomorphic semi-groups and the geometry of Banach spaces, Ann. of Math. (2) 115 (1982), $375-392$. 